\title{
AVALIAÇÃO DA DISTRIBUIÇÃO ESPACIAL DA MALÁRIA E DA LEISHMANIOSE TEGUMENTAR NO MUNICÍPIO DE TOMÉ-AÇU/PA
}

\author{
Raquel Soares Casaes Nunes ${ }^{1}$ \\ Roberta Soares Casaes ${ }^{2}$
}

\begin{abstract}
RESUMO
O município de Tomé-Açu/PA possui desenvolvimento voltado para a agricultura, mas em decorrência do desmatamento e do crescimento da cidade, a população tornou-se suscetível ao contato com vetores de Doenças Infecciosas e Parasitárias (DIPs). Em virtude das insuficientes informações sobre a distribuição espacial da ocorrência de DIPs na mesorregião do nordeste paraense e da possibilidade de novas informações que contribuam para a gestão ambiental dos municípios, busca-se através deste trabalho mapear a distribuição espacial de 2008 e 2018 sobre os casos registrados no município. Trata-se, portanto, de uma pesquisa quantitativa e qualitativa que correlaciona as DIPs Leishmaniose Tegumentar e Malária como desflorestamento. Os dados foram obtidos na Secretária de Saúde do município para a obtenção dos locais de ocorrência das doenças. Após o procedimento de importação dos dados, foram aplicados o estimador de Densidade de Kernel e o Índice Global de Moran para gerar mapas de 2008 e 2018 que evidenciam as diferentes densidades dos fenômenos e as áreas de maior risco epidemiológico, onde o uso e ocupação do solo são fatores predominantes para a epidemia de DIPs na região devido à ocupação humana nas proximidades de áreas recémdesflorestadas. De acordo com os números de casos registrados, a Leishmaniose Tegumentar foi a doença com maior incidência nos anos de 2008 e 2018. No entanto, a Malária teve um predomínio na área rural em 2008. Conclui-se que a metodologia utilizada pode contribuir para entendimento de onde devem ser intensificadas ações de vigilância e controle para a saúde da população.
\end{abstract}

Palavras-chave: SIG. Leishmaniose Tegumentar. Malária. Desflorestamento.

\begin{abstract}
The municipality of Tomé-Açu/PA has development geared to wards agriculture, but as a result of deforestation and the city's growth, the population has become susceptible to contact with vectors of Infectious and Parasitic Diseases (IPDs). Due to insufficient information on the spatial distribution of the occurrence of DIPs in the mesoregion of northeastern Pará and the possibility of new information that contributes to the environmental management of municipalities, this work seeks to map the spatial distribution of 2008 and 2018 on the cases registered in the municipality. It is, therefore, a quantitative and qualitative research that correlates the Cutaneous Leishmaniasis and Malaria IPDs with deforestation. The data were obtained from the municipality's health secretary to obtain the places where the diseases occurred. After the data import procedure, the Kernel Density estimator and the Global Moran Index were applied to generate maps for 2008 and 2018 that show the different densities of the phenomena and the areas of greatest epidemiological risk, where the use and occupation of the are predominant factors for the epidemic of IPDs in the region due to human occupation in the vicinity of newly deforested areas. According to the numbers of registered cases, Cutaneous Leishmania is was the disease with the highest incidence in the years 2008 and 2018. However, Malaria predominated in
\end{abstract}

\footnotetext{
${ }^{1}$ Graduada em Ciência Biológicas: Modalidade Microbiologia e Imunologia/UFRJ, Especialista em Segurança Alimentar /IFRJ mestre em Ciência de Alimentos/UFRJ e Doutora em Ciência de Alimentos/UFRJ, lecionou na Universidade Santa Úrsula e Universidade Salgado de Oliveira e tutora do SENAC RIO. Vinculada a projetos de pesquisas na área de Preservação de Alimentos com ênfase em segurança microbiológica dos alimentos de origem animal. Atualmente é docente da Universidade Federal Rural da Amazônia vinculada ao Instituto da Saúde e Produção Animal, com pesquisas em fungos e bactérias toxigênicas no cacau. E-mail: raquelcasaes@gmail.com.

${ }^{2}$ Mestre em Ciências de Alimentos UFRJ, Doutoranda em Biociências UNIRIO e Docente assistente do Curso de Nutrição UFRJ/Macaé. E-mail: betacasaes@gmail.com.
} 
the rural area in 2008. It is concluded that the methodology used can contribuited the understanding from where surveillance and control actions for the population's health could be intensified.

Keywords: GIS. Cutaneous, Leishmaniasis. Malaria. Deflorestation.

Data de submissão: 10.07 .2020

Data de aprovação: 06.08 .2020

\section{INTRODUÇÃO}

A Região Amazônica, por ser rica em minérios, açudes e por sua flora, recebeu grandes projetos de infraestrutura na década de 1980, proporcionando rápida expansão populacional associada a processos emigratórios e apenas no Estado do Pará encontram-se duas usinas hidrelétricas (MARINHO et al., 2016). O município de Tomé-Açu/PA apresenta desenvolvimento voltado para a agricultura e é a principal atividade econômica, tornando-se um dos mais importantes do estado, por apresentar diversidades nas atividades produtoras (HOMMA et al., 2018).

Em decorrência do desmatamento em áreas de floresta para agricultura e o crescimento da cidade, a população tornou-se mais suscetível ao contato com os vetores de doenças infecto parasitárias como Leishmaniose e Malária (PEREIRA; LOPES; NEVES, 2015).

A Leishmaniose é uma doença infecciosa crônica, causada pela Leishmaniose chassi, um protozoário pertencente ao complexo Leishmaniose donovani, sua transmissão se dá pela picada de várias espécies de flebotomíneos (BARRETO et al., 2011). A Leishmaniose Tegumentar americana é transmitida pelas múltiplas espécies de Leishmania e a forma mais simples da doença é a Leishmaniose cutânea localizada (LCL) (SILVEIRA et al., 2008). Apresenta uma ampla distribuição geográfica com diferentes aspectos climáticos e sociais, devendo-se ter uma atenção ao diagnóstico para o tratamento e a carência de saneamento básico para remediar a sua incidência (BARRETO et al., 2013).

Já a infecção da Malária resulta da picada do mosquito hematófago Anopheles darlingi (fêmea) infectado por Plasmodium falciparum, o mais patogênico, e o P. vivax. Apresentam alta prevalência na Amazônia, pois fatores geográficos e socioeconômicos da região facilitam a transmissão e dificultam o controle da proliferação do mosquito (BARRETO et al., 2011).

As distribuições geográficas das Doenças Infecciosas Parasitárias (DIPs), ocorrem principalmente em países em desenvolvimento, onde há uma relação entre fatores como ausência ou inadequação do saneamento básico, crescimento desordenado urbano e o desflorestamento, com a incidência dessas doenças (PEREIRA; ALVES-SOUZA; VALE, 2015). A água potável, um dos fatores responsáveis por promover saúde e qualidade essencial à vida, participa dessa distribuição, é evidente a associação entre proliferação de doenças e a falta de saneamento, já que doenças de veiculação hídrica são a segunda maior causa de morte na infância (MOURA; LANDAU; FERREIRA, 2016).

Nos estados amazônicos, a distribuição geográfica das DIPs é homogênea e atingem principalmente áreas com condições de habitação e de trabalho insatisfatórios, como projetos de colonização agrária e intensa migração da zona rural para periferia das cidades (RODRIGUES; LÚCIA, 2008).

Em geral, a noção de urbanização refere-se a mudanças no tamanho, densidade e heterogeneidade das cidades, tal nível de progresso teve algumas consequências negativas, como o volume de desmatamento para possibilitar atividades agroindustriais ou a extração de produtos básicos, o que contribuiu para diminuição das distâncias entre cidades e o aumento da mobilidade da população. Com isso, doenças anteriormente restritas às áreas rurais apareceram em áreas urbanas (BARRETO et al., 2011; WERNECK, 2008). 
Dentro desse contexto de urbanização, abordagens geograficamente espaciais são utilizadas frequentemente para determinar a epidemiologia da região em questão por meio de aplicação do processamento e análise das áreas geograficamente referenciadas. A partir de então, é possível compreender a disseminação de doenças e agravos à saúde, além de relacionar fatores patológicos e seus ambientes geográficos (BONFIM; MEDEIROS, 2014).

A análise da distribuição espacial de casos epidemiológicos, dentro de um mesmo município, é fundamental para a identificação de áreas de risco. Na Amazônia há uma combinação de fatores que favorecem o desenvolvimento do parasito e dificultam as medidas de prevenção e de controle que incluem características ambientais: o calor, a umidade e as coleções hídricas abundantes; aspectos antrópicos: desmatamento, ocupação de áreas indevidas, ausência de saneamento básico; e dificuldades para plena operacionalização de programas de controle dos parasitas, como inserção de inseticidas.

Essas desigualdades regionais e sociais podem ser identificadas através de informações epidemiológicas, através das análises dos locais de incidência das DIPs e das relações com as condições de habitação, alimentação e higiene precárias (ARAUJO, 2012). A constante modificação do ambiente destrói o habitat e desfaz o equilíbrio biológico que controla o número de exemplares existentes nestas populações, consequentemente altera a eco epidemiologia dos hospedeiros e a diversidade genética do parasito, fazendo com que os vetores busquem novas alternativas de sobrevivência (CARMO et al., 2016).

Em virtude das insuficientes informações sobre a distribuição espacial da ocorrência de Doenças Infecciosas e Parasitárias na mesorregião do nordeste paraense e da possibilidade de novas informações que contribuam para a gestão ambiental dos municípios, buscou-se através deste trabalho elaborar o mapeamento no intervalo de dez anos, de 2008 e 2018, sobre os casos registrados no município de Tomé-Açu/PA.

A aplicação dos Sistemas de Informação Geográfica (SIG) na pesquisa em saúde vem contribuir para a identificação de áreas geográficas e grupos da população que estão mais suscetíveis a adoecer ou morrer prematuramente, portanto, que necessitam de maior atenção, de forma preventiva (CRISTINA; VILLA, 2000a).

O uso de técnicas de análise espacial pode ser uma nova forma de avaliar o contexto e seus fatores de risco (RODRIGUES; LÚCIA, 2008) e de aperfeiçoar o planejamento de intervenções e monitoramentos seletivos conforme as reais necessidades de pequenas áreas (ARRAES; XIMENES, 1999).

Nesse sentido, o uso dos SIGs torna-se essencial para o trabalho, sendo importante para aplicação na área da saúde, destacando-se as análises de distribuição de endemias (SILVA et al., 2012). Espera-se, com a utilização dessas técnicas, fazer comparações e verificar possíveis tendências em torno dos dados obtidos.

Embora estudos epidemiológicos de análise espacial relacionados à incidência de casos de doenças infecciosas e parasitárias sejam frequentemente realizados (RODRIGUES; ESCOBAR; SOUSA-SANTOS, 2008; SILVA et al., 2017; VASCONCELOS; NOVO; DONALISIO, 2006), esses trabalhos concentram-se principalmente na região sul do país. No estado do Pará há poucos trabalhos e ainda não há nenhum estudo realizado no município de Tomé-Açu publicado.

Nesse contexto, o presente estudo propõe-se a contribuir com a gestão em saúde dos municípios, fornecendo a esses órgãos novos métodos de administração em saúde pública com o uso do SIG e a utilização de dados espaciais como instrumento efetivo na gestão ambiental e de saúde coletiva. 


\section{TECNOLOGIAS PARA ANÁLISES BIOGEOGRÁFICAS}

À medida que as sociedades avançam, tanto no que diz respeito às novas tecnologias quanto nas estruturas sociais, políticas e econômicas, elas se tornam cada vez mais complexas, estimulando que se procure compreender todas essas mudanças de maneira mais efetiva e rápida.

Este trabalho baseia-se em fundamentos importantes da geografia e em ferramentas tecnológicas para fins de entender as dinâmicas entre a sociedade e o meio ambiente, tendo como acometimento os efeitos dessa relação na saúde humana, como suporte para execução do trabalho temos: Sistemas de Informações Geográficas, Uso e Ocupação do Solo e Epidemiologia.

\subsection{SISTEMAS DE INFORMAÇÕES GEOGRÁFICAS}

Dentre as diversas tecnologias empregadas em análises espaciais, os SIGs são programas computacionais que permitem visualizar mapas geograficamente referenciados em conjunto com atributos de aspectos representados (CHIARAVALLOTI NETO, 2017). Estes sistemas também são definidos como sendo a fusão de técnicas e conceitos de ferramentas desenvolvidas pela informática, capazes de capturar, armazenar, processar dados e mostrar informações espaciais. A partir disso, o processo de construção do banco de dados para um SIG e a união de atributos é realizado com dados de campo, tais como o tipo de solo, a precipitação, imagens de satélite e fotografias aéreas que serão processados e manipulados posteriormente (DE ALMEIDA; MELO, 2011).

No âmbito da saúde, a aplicação dos SIGs agrega valor ao uso da identificação de áreas de risco econômico e socioambiental, nas quais essas populações estão suscetíveis às possíveis doenças parasitárias que podem levar a morte prematura e, por conta disto, demandam atenção especial no sentido de prevenir e promover a saúde coletiva (CRISTINA; VILLA, 2000b). Assim, para que sejam realizadas análises de dados, se torna necessário possuir a localização geográfica dos locais de ocorrência das doenças e associar informações gráficas às bases de dados de saúde (SKABA et al., 2007).

\subsection{USO E OCUPAÇÃO DOS SOLOS}

Devido ao uso de imagens de satélite e radar, as novas tecnologias em sensoriamento remoto permitem aprofundar pesquisas de investigação espacial e trazem benefícios, como as análises em larga escala seguido de maior precisão, principalmente quando relacionados aos problemas ambientais humanos. Como exemplo disso, podem-se citar alguns dos diversos fatores relacionados ao uso do solo que favorecem a reprodução do mosquito da dengue, dentre eles, a urbanização crescente e sem planejamento que resulta em regiões com altos índices demográficos, falta de água, pouca limpeza urbana, o tráfego elevado de pessoas e, principalmente, a não efetividade do combate ao vetor (COSTA et al., 2018).

Ainda nesse contexto, observa-se que a distribuição espacial da Malária na Amazônia não é igualitária. Ela afeta, sobretudo, populações instaladas em ambientes onde a habitação é desordenada e com níveis de pobreza elevados, além de locais onde há exploração de recursos naturais em projetos de assentamento (RODRIGUES; ESCOBAR; SOUSA-SANTOS, 2008).

Além disso, os estudos epidemiológicos que antes eram realizados apenas com dados estatísticos, números de casos e pesquisas documentais como análises de prontuários, agora passam a ter análises espaço-temporais usando sensoriamento remoto como ferramenta importante para fins de controle e planos estratégicos sobre áreas afetadas por determinada doença infecto parasitária. 


\subsection{EPIDEMIOLOGIA}

A epidemiologia faz parte do movimento da saúde pública e da saúde coletiva e tem como atribuições gerar conhecimentos, informações e tecnologias que possam ser utilizadas na formulação, prevenção e controle dos problemas, desenvolvendo condições de saúde pública (BARATA, 2013). A interação entre padrões de saúde/doença com fatores demográficos, econômicos e sociais influenciam nas complexas mudanças na transição epidemiológica. Diante dessas constantes mudanças, o não conhecimento dos aspectos ambientais, ecológicos do parasita, clínicos e laboratoriais, impossibilitam a implementação de medidas que evitem a proliferação da doença (PEREIRA; ALVES-SOUZA; VALE, 2015).

Aliás, estudos para definir os riscos epidemiológicos de determinada região, são de extrema importância para indicar áreas prioritárias para controle de doenças como, por exemplo, a Leishmaniose e a Malária. Com base em mapas que consideram o risco de ocorrência da endemia e no nível de ocupação espacial das áreas geográficas, tem-se uma importante ferramenta para serviços de vigilância e controle (RODRIGUES; ESCOBAR; SOUSA-SANTOS, 2008; STATE et al., 2017), ou seja, por meio da utilização de ferramentas de sensoriamento, é possível montar um padrão espacial de infecção, pois a progressão de tais doenças dependem das condições ecológicas que facilitam a dispersão de mosquitos transmissores (COSTA et al., 2018).

No que dizem respeito às leishmanioses (visceral e tegumentar), segundo o Sistema de Informações de Agravos de Notificação (SINAN), elas apresentam elevadas prevalências na Região Norte (área Amazônica). Ademais, seu perfil epidemiológico tem sofrido alterações e vem expandindo sua área de ocorrência, geralmente associada às modificações ambientais provocadas pelo homem, fazendo parte do grupo de Doenças Tropicais Negligenciadas (DTN) (SILVA et al., 2017).

A Malária, por sua vez, em 1976 apresentava menos de 100.000 casos por ano, já nos anos seguintes (a partir de 1999), houve elevação do número de casos da doença em função da ocupação desordenada da Região Amazônica, resultante da implantação de projetos de colonização e mineração sem a necessária estrutura de saúde para atender a população. No entanto, após amplo processo de mobilização de forças multissetoriais pelo Ministério da Saúde, observou-se o declínio no número de casos em 2016 (BRAZ; BARCELLOS, 2018).

\section{MATERIAL E METODOLOGIA}

\subsection{COLETA DE DADOS}

As informações necessárias para a realização deste trabalho foram obtidas a partir do banco de dados da Secretaria Municipal de Saúde do Município de Tomé-Açu, a qual teve como fonte o Sistema de Informações de Agravos e Notificação (SINAN) para Leishmaniose Tegumentar, e do Sistema de Informações de Vigilância Epidemiológica (SIVAP) para os dados da Malária que repassaram os números de casos e os locais de ocorrência registrados nos anos de 2008 e 2018.

Além desses dados, foram adquiridos shape files do local de interesse, município de Tomé-Açu, no banco de dados do Instituto Brasileiro de Geografia e Estatística (IBGE). O shape file é um tipo de arquivo vetorial utilizado em Sistemas de Informações Geográfica que armazena posição, forma e feições geográficas, simulando a forma real da Terra e de suas regiões. Ele é o principal componente responsável pelo recorte dos limites municipais da área de interesse para o estudo, utilizando o sistema de coordenadas projetadas para alocação dos pontos de infecção ou caso de doenças. Por sua vez, as coordenadas projetadas foram 
coletadas através do software Google Earth de acordo com os dados repassados pela Secretaria Municipal de Saúde.

Também foram coletadas imagens de satélites do banco de dados do centro de pesquisas espaciais da United States Geological Survey (USGS) dos dias 13 de julho/2008 do satélite Landsat 5 sensor TM, e 23 de junho/2018 do satélite Landsat 8 sensor OLI. Faz-se necessário pontuar ainda que o sistema de projeção cartográfica utilizado neste trabalho foi o UTM (Universal Transversa de Mercartor), sendo esse um modelo matemático que representa a forma da Terra no fuso 22S e Datum SIRGAS 2000.

\subsubsection{PROCESSAMENTO DAS INFORMAÇÕES COLETADAS}

Após a coleta dos pontos em graus decimais, padrão das coordenadas projetadas, eles foram organizados em planilha para posteriormente serem submetidos ao software Arc GIS 10.5 para transformação em pontos de ocorrência dentro do mapa já configurado. Os números de casos coletados também foram organizados em planilha para geração dos gráficos. Depois da tabulação dos dados, verificaram-se todos os locais de ocorrência de DIPs dentro do município. Assim, os pontos de interesse foram agrupados de modo que a cada área fosse empregado o número de casos fornecidos pela Secretaria Municipal de Saúde.

As coordenadas de cada região afetada pelas doenças foram tabuladas para, em seguida, serem transformadas em arquivo de dados X e Y no formato shape file. Esse arquivo foi aplicado ao mapa e logo após submetido à ferramenta de Densidade de Kernel do Acr GIS 10.5. Através desta ferramenta foram gerados os mapas de densidade das DIPs dos anos de 2008 e 2018, nos quais é possível visualizar a dinâmica dos padrões de densidade dos eventos considerados no estudo. Além disso, a Densidade de Kernel ainda faz a contagem de todos os pontos dentro de um limite de influência em conjunto do tratamento estatístico para verificar a autocorrelação espacial entre os locais de ocorrência e a densidade de casos.

De acordo com Silver (1986), o estimador de Densidade de Kernel é definido pela Equação 1 abaixo:

$$
\hat{f}(k)=\frac{1}{n h^{2}} \sum_{i=1}^{n} k\left\{\frac{x-x_{i}}{h}\right\}
$$

Onde, $n$ é o número de pontos observados, $h$ é a largura da banda, $k$ é a Função de Kernel, $x$ é a coordenada vetorial que representa a distância entre os pontos e $x_{i}$ é o vetor da iésima coordenada representando cada ponto observável em relação ao estimado.

Após a aplicação da Densidade de Kernel, mapas foram gerados por meio da interpolação de pontos, calculados a partir de pontos (vizinhos) mais próximos. Em seguida, foram adicionadas seis classes de temperatura, sendo de 0-100 para as temperaturas mais baixas, com menor risco epidêmico, até 500-1.000 para as áreas com a maior taxa de ocorrência de risco epidêmico dentro da distribuição espacial.

\subsection{TRATAMENTO ESTATÍSTICO (ÍNDICE GLOBAL DE MORAN)}

Para avaliar a autocorrelação espacial entre os pontos de ocorrência e o local de estudo, município de Tomé-Açu/PA, foi aplicado o tratamento estatístico de Índice Global de Moran, que se trata de um modelo matemático e uma ferramenta existente dentro do Arc Map 
do Arc GIS 10.5. O tratamento estatístico foi aplicado sobre todos os dados (pontos) das doenças Leishmaniose Tegumentar e Malária.

Após a coleta e aplicação da Densidade de Kernel sobre os pontos de ocorrência, as mesmas coordenadas foram empregadas dentro da ferramenta estatística para fins de se obter a significância dos dados quanto a autocorrelação espacial. Um índice com o valor maior de 0,05 ( $p>0,05$ ) demonstra que os dados não são significativos.

A obtenção dos resultados foi efetuada de modo quantitativo no sentido de que os índices obtidos através da autocorrelação espacial demonstram agrupamentos, aleatoriedade ou dispersão dos dados pontuais analisados (locais de ocorrência), e também de modo qualitativo, pois os resultados do tratamento estatístico aplicado sobre todos os dados pontuais foram relacionados com a interpretação visual dos mapas de Densidade de Kernel, sendo atribuído a variável (uso e ocupação do solo) dentro da análise qualitativa.

\subsection{PROCESSAMENTO DIGITAL DAS IMAGENS (PDI)}

As etapas de processamento e classificação das imagens foram realizadas no software Arc Gis versão 10.5.0. Para possibilitar a classificação de forma mais eficiente foi realizada a composição em falsa cor natural utilizando as bandas R(5), G(4), B(3) do satélite Landsat 5 e $\mathrm{R}(6), \mathrm{G}(5), \mathrm{B}(4)$ do satélite Landsat 8, seguida da correção geométrica das imagens do sensor TM com a obtenção de 28 pontos, tomando como base a imagem em falsa cor natural do sensor OLI.

Para a realização da classificação, as imagens passaram pelo pré-processamento, sendo importante para melhorar a qualidade dos dados, fazendo uso das técnicas de ajuste da resolução das imagens, correção geométrica e registro (EASTMAN, 2006).

A escala adotada para a classificação foi de 1:100.000, onde a interpretação visual foi aplicada de acordo com comportamento espectral dos pixels, sendo caracterizada por três regiões distintas: visível, infravermelho próximo e infravermelho médio (MOREIRA, 2001). O método de classificação utilizado foi a de Máxima Verossimilhança MAXVER, seguida da distribuição de polígonos e identificação de cada um de acordo com a classe atribuída. $\mathrm{O}$ classificador MAXVER é utilizado nos principais SIGs de processamento digital de imagem, sendo considerado um dos melhores e mais utilizados (BARBOSA et al., 2011).

O município de Tomé-Açu/PA, área selecionada para este estudo, localiza-se no estado do Pará e faz parte da mesorregião do nordeste paraense, abrangendo uma área territorial de $5.145,361 \mathrm{~km}^{2}$, com uma população de 56.518 pessoas e densidade demográfica de $10,98 \mathrm{hab} / \mathrm{km}^{2}$ de acordo com o IBGE de 2010 .

\section{RESULTADOS E DISCUSSÃO}

Os casos de Leishmaniose Tegumentar e Malária tiveram o total de 886 casos registrado pelo SINAM-PA nos anos de 2008 e 2018. Destes, 642 foram excluídos por se tratarem de casos descartados (negativos), sendo considerados apenas 244 casos confirmados pela Secretaria de Saúde do município, como mostra a Tabela 1.

Tabela 1 - Número de casos confirmados e registrados de DIPs em Tomé-Açu (2008-2018)

\begin{tabular}{rrr|rrrr}
\hline \multicolumn{3}{c|}{ CASOS REGISTRADOS } & \multicolumn{3}{c}{ CASOS CONFIRMADOS } \\
Ano & L. Tegumentar & Malária & Ano & L. Tegumentar & Malária \\
\hline 2008 & 139 & 637 & 2008 & 121 & 69 \\
2018 & 51 & 59 & 2018 & 51 & 3 \\
Total & 190 & 696 & Total & 172 & 72 \\
\hline
\end{tabular}

Fonte: Elaborada pelos autores. 
Para melhor visualização dos locais de ocorrência, a Figura 1 a seguir traz o mapa temático do município de Tomé-Açu/PA, evidenciando nele os locais com paisagem natural e antrópica. Nele, atribuíram-se seis classes: pasto, vegetação secundária, vegetação primária, espelho d'água (rios, lagos, igarapés), solo exposto e área urbana. A vegetação primária, considerada as matas ciliares e florestas nativas, e a secundária, aquela na qual houve impacto antrópico ou natural.

De acordo com o cálculo geométrico das classes, o tamanho das áreas ocupadas é de $32,6 \%$ para a classe pasto, $16,1 \%$ de vegetação secundária, 50,8\% de vegetação primária e apenas $0,1 \%$ de espelho d'água, $0,2 \%$ de solo exposto e $0,2 \%$ de área urbana.

Figura 1 - Mapa Temático do Município de Tomé-Açu/PA

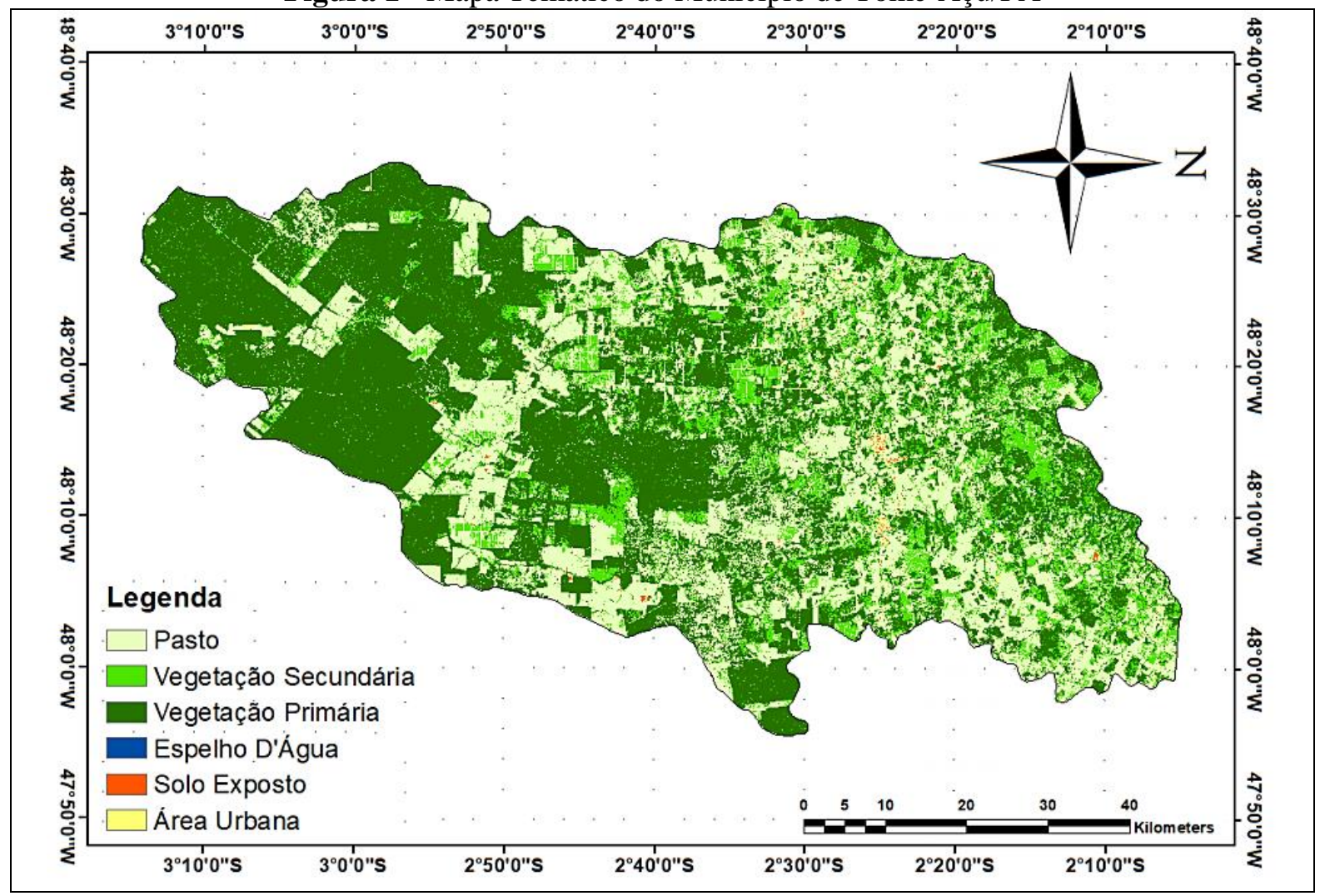

Fonte: Autores, 2020.

Nos mapas da distribuição espacial da Leishmaniose Tegumentar, é possível observar que os pontos mais quentes são próximos à sede municipal de Tomé-Açu/PA, ao distrito de Quatro-Bocas e à Forquilha. A sede municipal apresentou valores na faixa de 500-1000 nos dois anos analisados, enquanto que o distrito de Quatro-Bocas entre 300-400 em 2008 e 2018 dentro da escala de densidade. Por sua vez, o distrito de Forquilha manteve o mesmo valor de densidade, entre 400-500, como apresentado na Figura 2.

No mapa do ano de 2018, verificou-se o prolongamento da distribuição espacial da Leishmaniose Tegumentar na região da PA 140, onde os casos da doença afetaram principalmente as comunidades de Água Branca e do KM 40. No ano de 2008, a distribuição espacial próximo à vila Forquilha se deu por conta das comunidades Mariquita e Jamic, como se observa na imagem direita da Figura 2.

Desse modo, verificou-se pouca mudança nos padrões analisados da distribuição espacial da Leishmaniose Tegumentar entre os anos de 2008 e 2018, com uma diferença maior apenas em uma parte da região da PA 140 em direção ao norte do município no ano de 2018. 
Os locais de maior concentração de casos no ano de 2018 foram: Ramal do Areial, Ramal Santa Rosa, Tropicalia, região da Jamic e Forquilha, totalizando 23 dos 51 casos confirmados. Em 2008 os locais com mais casos eram: comunidade Rural Calmaria, Jamic, bairro Tabom, Canindé, Forquilha, Centro de Tomé-Açu, Sempre Alegre e Novo Horizonte em Quatro-Bocas com o total de 35 dos 121 casos confirmados.

Figura 2 - Mapas de Densidade de Kernel para os casos de Leishmaniose Tegumentar (2008-2018)

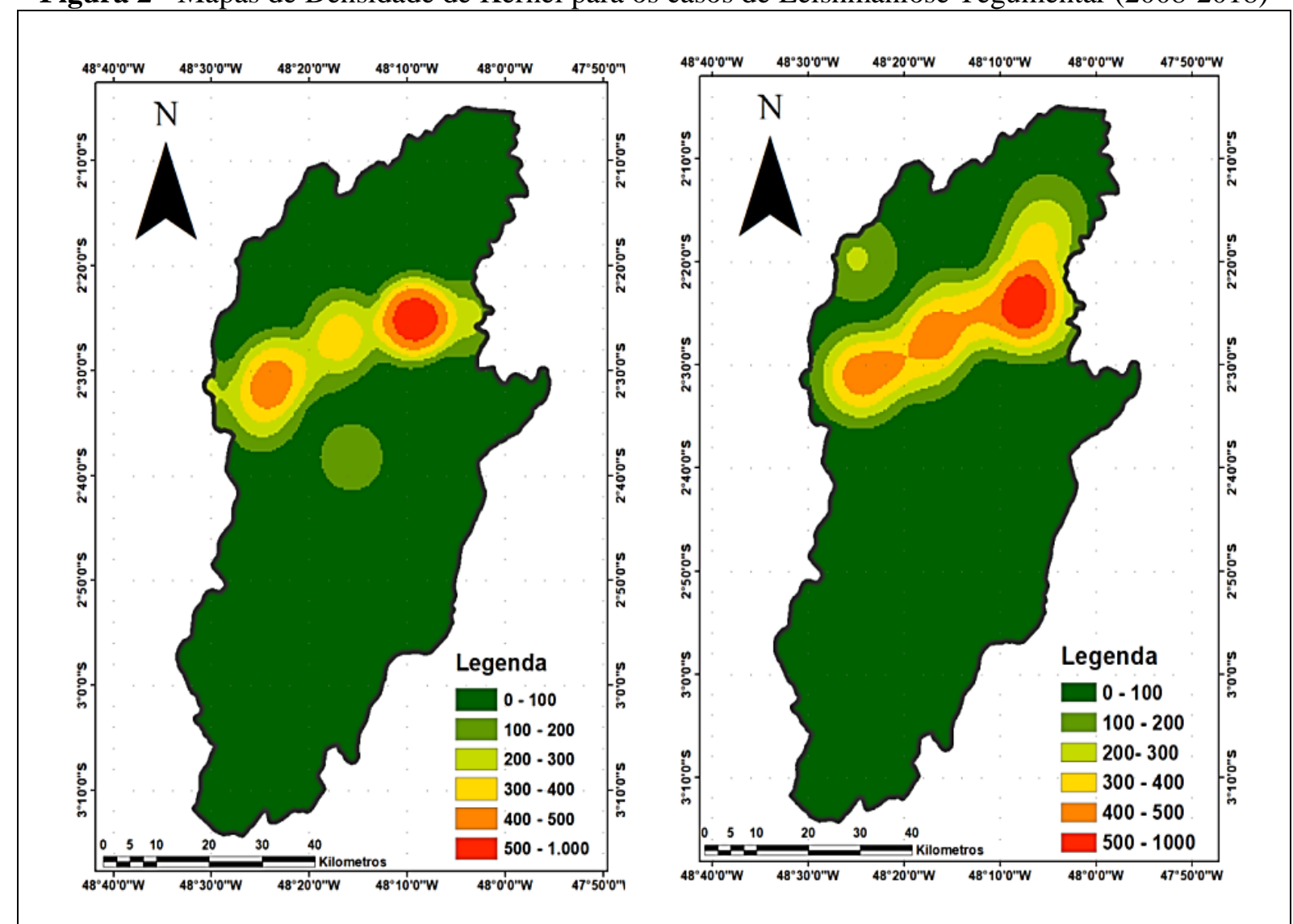

Fonte: Autores, 2020.

Verificou-se ainda a presença de autocorrelação espacial dos casos de Leishmaniose Tegumentar, no sentido de que o tratamento estatístico aponta o agrupamento com Índice de Moran igual a 0,43 e p valor igual a 0,00. Considerando o p valor $<0,05$, então os dados para autocorrelação espacial são significativos. Dado o valor de z-score: 6,255, há uma probabilidade inferior a $1 \%$ que esse padrão agrupado pode ser resultado de chance aleatória.

No entanto, a Malária apresentou o padrão de distribuição espacial diferente quando comparada à Leishmaniose Tegumentar, onde em 2008 houve uma alta densidade com escala no intervalo de 500-1000 na região da zona rural Manga Larga, decaindo constantemente de acordo com o distanciamento do foco epidêmico, o número total de 42 casos, na parte norte do mapa, a densidade está entre 100-200 e 200-300, cobrindo as áreas urbanizadas e comunidades vizinhas como: colônia Santa Maria, comunidade Socorro, KM 40 e KM 23, totalizando 27 casos, como apresentado na imagem esquerda da Figura 3.

O ano de 2018 proporcionou a distribuição com os casos registrados centralizados na área urbana, sendo um caso na Sede, um no distrito de Quatro-Bocas e o outro na comunidade rural Socorro, como apresentado na imagem direita da Figura 3. Além da região da comunidade Manga Larga, em outros locais houve concentração de Malária, entretanto, o número das ocorrências foi menor. Os locais foram: distrito de Quatro-Bocas, com três casos 
confirmados; comunidade Vila Nova, Vera Cruz, Diamante, Santa Maria e Água Branca totalizando 12 casos registrados, porém não confirmados.

Nas demais áreas como Ramal do Meio, Jutaí, Glória e Marupiara, que são comunidades rurais, houve apenas casos isolados registrados, totalizando apenas cinco casos, um em cada região.

Figura 3 - Mapas de Densidade de Kernel para os casos de Malária (2008-2018)

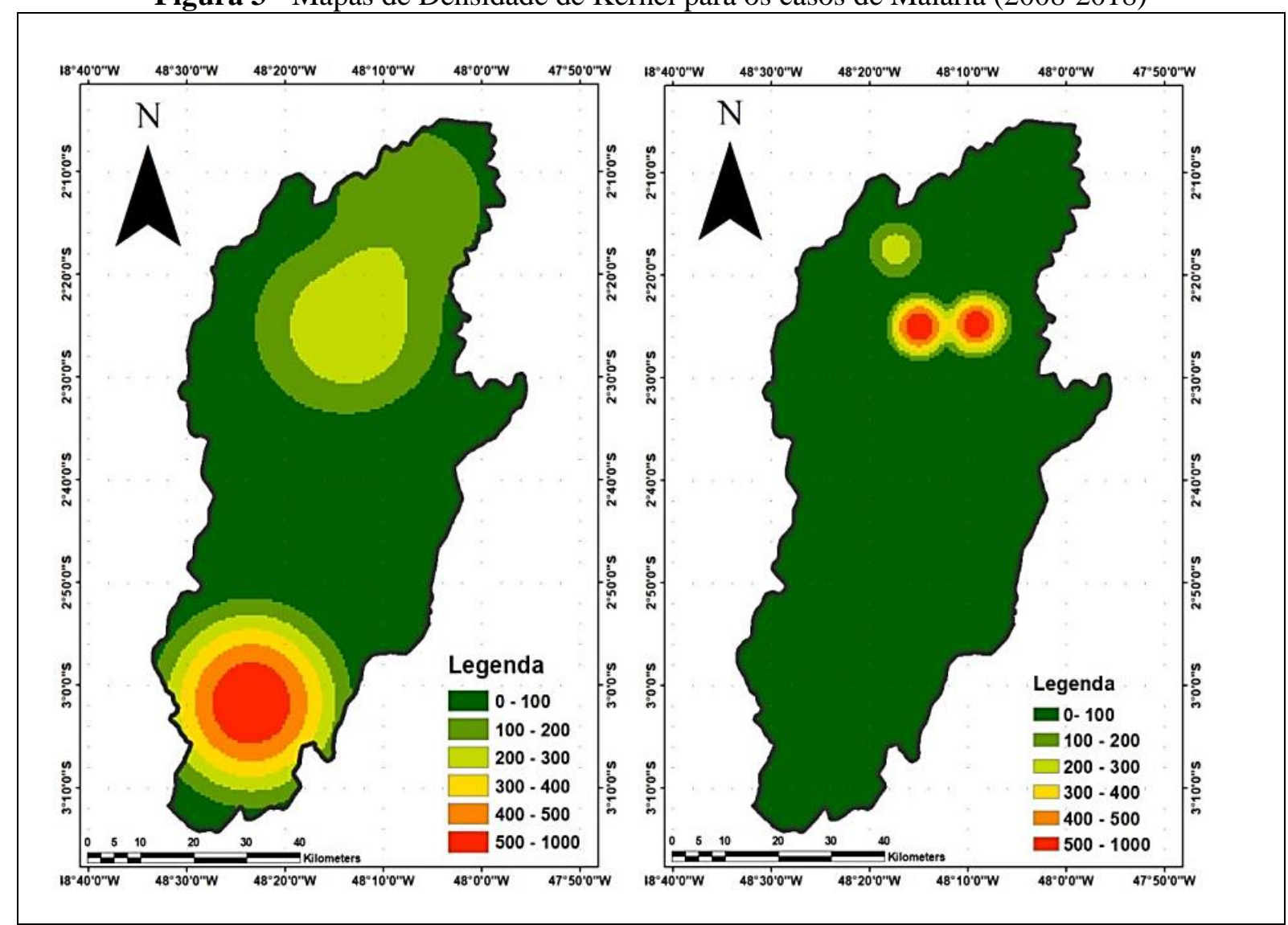

Fonte: Autores, 2020.

Para os casos de Malária, o Índice de Moran apresentou o valor de 0,39 e p valor igual a 0,01 , com o resultado foi $\mathrm{p}$ valor $<0,05$, os dados demonstraram-se significantes para o estudo. Com o z-score: 2,44 , há menos que $5 \%$ de probabilidade de que esse padrão de agrupamento seja resultado ao acaso.

A partir do exporto acima, o presente estudo, pioneiro para o município de ToméAçu/PA, obteve informações essenciais referentes ao perfil epidemiológico das doenças infecciosas e parasitárias causadas pela Leishmaniose Tegumentar e pela Malária. Os resultados obtidos pela análise do banco de dados do SINAM-PA e pela visualização da distribuição espacial dos casos, que apresentam uma densidade nos mapas em regiões próximas à área urbana e dentro da área urbana, podem servir como base para resolução de condutas da vigilância epidemiológica estadual.

Observa-se ainda que em todos os mapas gerados, as DIPs concentram-se, principalmente, na região norte do município, em todos os anos e locais analisados é visível esse mesmo padrão de distribuição. Desse modo, com o mapa temático gerado a partir da classificação supervisionada, é possível verificar a geomorfologia da paisagem no que diz respeito às áreas com maior cobertura vegetal densa, como vegetação primária e as de menor cobertura vegetal, como a vegetação secundária, pasto, área urbana e solo exposto. 
A gestão dos recursos naturais demanda uma caracterização constante, além do monitoramento contínuo, para fins de aproveitar e melhorar o uso dos recursos com foco no controle e uso racional adequado (GAROFALO et al., 2015). Além disso, o controle de epidemias também pode ocorrer através do uso do SIG, como na Figura 1 que proporciona uma visão ampla e apresenta a situação das áreas verdes no município, mais preservadas ou exploradas com mais intensidade, além das regiões de pasto ou solo exposto.

O processo de monitoramento dos recursos naturais é importante no sentido de controle epidemiológico, como mostra o trabalho de Silva et al. (2017), no qual os casos de Leishmaniose Visceral situados nas áreas rurais do município de Abaetetuba/PA, estão fortemente relacionados às condições ambientais que propiciam o aparecimento da doença, como o processo de desmatamento e ocupação desordenada do território pelas pessoas.

Nesse sentido, nota-se a importância de relacionar as condições ambientais aos problemas de saúde coletiva presentes em determinadas regiões, além de que a elaboração de mapas temáticos contribui como um instrumento de extrema importância para a visualização de modo mais amplo, pois através dele é possível observar as áreas naturais e ocupadas em larga escala (NASCIMENTO; MORAES, 2019).

A análise espacial da distribuição da ocorrência de casos de Malária em 2008 registrou 69 casos, sendo 42 na zona rural Manga Larga, localizada a $143 \mathrm{~km}$ da sede municipal. Esse predomínio pode ser explicado pelo uso indevido do solo, como desmatamento de áreas extensas, grandes migrações com formação de aglomerações desprotegidas e a falta de estrutura da saúde (BRAZ; BARCELLOS, 2018).

Além disso, Braz e Barcellos (2018) realizaram análise da distribuição dos municípios em 2008 e, de acordo com a classificação da variação da incidência da Malária, verificaram os municípios que estavam em processo de eliminação da doença. Segundo os autores, o município de Tomé-Açu/PA participou desse processo e apresentou o Índice Parasitário Anual (IPA) de 1 caso para cada 1.000 habitantes. No presente estudo, com a avaliação da distribuição espacial da frequência de novos casos, é possível demonstrar a eficácia no processo de redução dos casos da Malária, que em 2008 apresentaram 69 casos confirmados, decaindo para 3 casos em 2018.

Diferentemente das outras doenças, a Leishmaniose apresenta padrões epidemiológicos extremamente diversos, não depende apenas de uma região geográfica distinta e está mais relacionada com fatores socioculturais, como ocupação de áreas recentemente desmatadas (COSTA, 2005).

Assim como a análise realizada por Catete et al. (2017), nos anos de 2007 a 2017, no município de Barcarena/PA, referente a distribuição espacial dos casos de Leishmaniose, demonstraram a ocorrência na extensão das áreas degradadas $\left(24,86 \mathrm{~km}^{2}\right)$, concentrada em áreas urbanas, em seguida os autores observam um aumento de áreas urbanas proporcional aos casos de Leishmaniose, corroborando a associação de modificações sociais e ambientais com o aumento dos casos.

De acordo com o cálculo geométrico das classes, o pasto ocupa 32,6\% do solo no município, essas modificações no habitat dos flebotomíneos para criação de animais para consumo, influenciam o vetor da Leishmania a ir ao ambiente domiciliar para se alimentar (SERPA et al., 2017). O predomínio dessa doença em áreas urbanas ocorre devido aos locais onde os flebotomíneos se desenvolvem sofrerem com a falta de saneamento adequado, visível no perímetro da cidade, pois os mosquitos de flebotomíneos preferem águas lentas, sombreadas e com matéria orgânica para depositar seus ovos (SERPA et al., 2017).

A Região Norte inclui os estados mais afetados com doenças relacionadas ao saneamento ambiental inadequado, entre as doenças está a Malária que apesar de não ser transmitida pela água está estritamente relacionada a ela. Uma vez que seus vetores transmissores de Malária dependem da água para se desenvolverem. E, levando em 
consideração os dados do IBGE de 2010, o município de Tomé-Açu/PA possui apenas 2,6\% de Saneamento Adequado e apenas 8 estabelecimentos de saúde. Esses fatores favorecem a questão da disseminação de DIPs no município (MOURA; LANDAU; FERREIRA, 2016).

\section{CONSIDERAÇÕES FINAIS}

Com este trabalho conclui-se que os métodos utilizados nele, como o mapeamento das áreas de maior densidade de determinadas doenças no município de Tomé-Açu/PA, bem como as características da paisagem nos locais acometidos, podem contribuir para o entendimento de onde devem ser intensificadas ações de vigilância e controle para melhorar a saúde da população com atividades preventivas.

Além disso, outra contribuição advinda deste trabalho se dá pelo estabelecimento de pontos estratégicos para verificação das áreas de influência das DIPs. Uma vez que os pontos estratégicos estão sugeridos nas diretrizes nacionais para a prevenção e controle de epidemias de doenças infecciosas e parasitárias, esta abordagem pode ser utilizada em outros municípios, com o intuito de saber se os pontos estratégicos vão influenciar ou não na ocorrência das doenças. Caso exista o predomínio de DIPs próximos aos pontos estratégicos, a prefeitura pode reforçar a fiscalização e remoção de criadouros para os vetores de Leishmaniose Tegumentar e Malária naqueles locais.

Observa-se ainda que apenas os dados do SINAM não foram suficientes para determinar a epidemiologia de determinado local, pois eles apresentam o total de casos registrados sem apresentar os casos positivos, sendo necessário outros dados para completar sua significância. Dessa forma, devem-se validar os casos na Secretaria de Saúde do município, assim como foi realizado no presente estudo, a fim de se obter o número de casos confirmados.

De acordo com os resultados deste estudo, as DIPs analisadas possuem forte correlação espacial entre os locais de ocorrência, levando em consideração o padrão de agrupamento que se concentra principalmente nas áreas rurais com atividade pecuária (pasto), como mostra o mapa temático e nas áreas urbanizadas.

Por fim, se faz necessário elucidar que há algumas limitações com relação à abordagem e métodos utilizados neste trabalho, já que outros fatores também podem influenciar na incidência das doenças, dentre eles os fatores climáticos como umidade, índice pluviométrico e temperatura que podem estar relacionados com a proliferação. Logo, os resultados obtidos no presente trabalho ${ }^{3}$ podem ser complementados com futuros estudos sobre a influência desses fatores.

\section{REFERÊNCIAS}

ARAÚJO, José Duarte de. Polarização Epidemiológica no Brasil. Epidemiologia e Serviços de Saúde, Brasília, v. 1, n. 2, dez./jan. 2012. Disponível em: http://scielo.iec.gov.br/pdf/ess/v21n4/v21n4a02.pdf. Acesso em: 24 maio 2019.

BARATA, Rita Barradas. Epidemiologia e Políticas Públicas. Revista Brasileira de Epidemiologia, v. 16, n. 1, p. 3-17, 2013. Disponível em:

\footnotetext{
3 Agradecimentos aos discentes de graduação da Universidade Federal Rural da Amazônia: Leandro Nascimento, Maria Letícia Marques pela colaboração no presente estudo com análises de georreferenciamento e o discente Esteferson Santana Quadros pela execução dos dados científicos obtidos do DATASUS.
} 
https://www.scielo.br/pdf/rbepid/v16n1/1415-790X-rbepid-16-01-0003.pdf. Acesso em: 24 maio 2019.

BARBOSA, Ana Paula et al. Comparação de métodos de Classificação de Imagens na Identificação de Áreas Cultivadas com Citros. Energia na Agricultura, Botucatú, v. 26, n. 3 p. 14-25, 2011. Disponível em:

http://revistas.fca.unesp.br/index.php/energia/article/view/270. Acesso em: Acesso em: 24 maio 2019.

BARRETO, Maurício et al. Sucessos e Fracassos no Controle de Doenças Infecciosas no Brasil: o contexto social e ambiental, políticas, intervenções e necessidades de pesquisa.

Saúde no Brasil, v. 3, p. 47-60, 2011. Disponível em:

http://bvsms.saude.gov.br/bvs/artigos/artigo_saude_brasil_3.pdf. Acesso em: 24 maio 2019.

BONFIM, Cristina; MEDEIROS, Zulma. Epidemiologia e Geografia: dos primórdios ao geoprocessamento. Revista Espaço para a Saúde: revista do Programa de Pós-Graduação em Ensino nas Ciências da Saúde da Faculdade Pequeno Príncipe, Londrina, v. 10, n., p. 5362, dez. 2014. Disponível em:

https://www.agbbauru.org.br/publicacoes/revista/anoXXII_1/agb_xxii_1_web/Rev_AGB_dez 2018-completa.pdf. Acesso em: 24 maio 2019.

BRAZ, Rui Moreira; BARCELLOS, Christovam. Análise do processo de eliminação da transmissão da malária na Amazônia brasileira com abordagem espacial da variação da incidência da doença em 2016. Epidemiologia e Serviços da Saúde, Brasília, v.27, n.3, p. 01-13. 2018. Disponível em:

https://www.scielo.br/scielo.php?script=sci_abstract\&pid=S2237-

96222018000300303\&lng=en\&nrm=iso\&tlng=pt. Acesso em: 24 maio 2019.

CARMO, et al. Soroepidemiologia da Infecção pelo Toxoplasma gondii no Município de Novo Repartimento, Estado do Pará, Brasil. Pan-Amazônia da Saúde, Ananindeua, v. 7, n. 4, p. 79-87, 2016. Disponível em:

http://scielo.iec.gov.br/scielo.php?script=sci_arttext\&pid=S2176-62232016000400079.

Acesso em: 24 maio 2019.

CATETE, Clístenes Pamplona et al. Geotecnologias aplicadas ao monitoramento de áreas degradadas e sua relação com casos de Leishmaniose, Barcarena (Pa), Brasil. In: SIMPÓSIO NACIONAL RECUPERAÇÃO DE ÁREAS DEGRADADAS, 2017, Curitiba. Anais [...]. Curitiba: Revendo princípios / validando conceitos. Sobrade, 2017. p. 1-12. Disponível em: http://www.sobrade.com.br/download/Anais\%20SINRAD_edicao\%20completa.pdf. Acesso 24 maio 2019.

CHIARAVAllOTI-NETO, Francisco. O Geoprocessamento e Saúde Pública. Arquivos de Ciências da Saúde1: revista da Faculdade de Medicina de São José do Rio Preto (FAMERP), São Paulo, v. 23, n. 4, p. 01-02, fev. 2017. Disponível em: http://www.cienciasdasaude.famerp.br/index.php/racs/article/view/661. Acesso em: 24 maio 2019.

COSTA, Marília Millena Remígio da. et al. Dengue: aspectos epidemiológicos no município de Salgueiro do Sertão Pernambucano, Brasil. Brazilian Journal of health Review, Curitiba, n.1 p. 260-266, jul./set. 2018. Disponível em: 
https://www.brazilianjournals.com/index.php/BJHR/article/view/678/577. Acesso em: 24 maio 2019.

COSTA, Jackson Mauricio Lopes. Epidemiologia das Leishmanioses no Brasil. Gazeta Médica da Bahia, n. Ic, p. 3-17, jan./jun. 2005. Disponível em:

https://www.arca.fiocruz.br/bitstream/icict/9808/2/Costa\%20JML\%20Epidemiologia\%20das \%20leishmanioses....pdf. Acesso 24 maio 2019.

DE ALMEIDA, Fabiane Ferreira.; MELO, Sérgio. Phytoplankton Community Structure in an Amazon Floodplain Lake (Lago Catalão, Amazonas, Brazil). Neotropical Biology and Conservation, v. 6, n. 2, p. 112-123, 2011. Disponível em:

http://revistas.unisinos.br/index.php/neotropical/article/view/nbc.2011.62.06. Acesso 24 maio 2019.

EASTMAN, Ronald. Idrisi 15: The Andes Edition. Worcester, MA: Clark University, 2006.

GAROFALO, Danilo Francisco Trovo et al. Análise Comparativa de Classificadores Digitais em Imagens do Landsat-8 Aplicados ao Mapeamento Temático. Pesquisa Agropecuária Brasileira, Brasília, v. 50, n. 7, p. 593-604, jul. 2015. Disponível em: https://www.scielo.br/pdf/pab/v50n7/1678-3921-pab-50-07-00593.pdf. Acesso em: Acesso 24 maio 2019.

HOMMA, Alfredo Kingo Oyama et al. Pequenos Produtores de Tomé-Açu e Viseu, Pará: da "Agricultura de Toco" a SAFS, Uma Mudança Possível?. In: CONGRESSO DA SOCIEDADE BRASILEIRA DE ECONOMIA, ADMINISTRAÇÃO E SOCIOLOGIA RURAL, 56., 2018, Campinas. Anais [...]. Brasília, DF: SOBER, 2018. Disponível em: https://www.alice.cnptia.embrapa.br/handle/doc/1094569. Acesso em: Acesso 24 maio 2019.

INSTITUTO BRASILEIRO DE GEOGRAFIA E ESTATÍSTICA. Censo Demográfico 2010. Disponível em: https://cidades.ibge.gov.br/. Acesso em: 19 jun. 2019.

LIMA, Sandra Souza et al. Análise Espacial da Tuberculose em Belém, estado do Pará, Brasil. Revista Pan-Amazônica de Saúde, Ananindeua, n. 2, p. 57-65, nov./dez. 2017. Disponível em: http://revista.iec.gov.br/submit/index.php/rpas/article/view/399/308. Acesso em: 24 maio 2019.

MARINHO, Jamille Salim et al. Original Doenças Infecciosas e Parasitárias por Veiculação Hídrica e Doenças Respiratórias em Área Industrial, Norte do Brasil. Caderno de Saúde Coletiva, Rio de Janeiro, n.4, out./dez. 2016. Disponível em: https://www.scielo.br/scielo.php?pid=S1414-462X2016000400443\&script=sci_arttext. Acesso em: 24 maio 2019.

MOURA, Larissa; LANDAU, Elena Charlotte; FERREIRA, Adriana de Melo. Doenças relacionadas ao saneamento ambiental inadequado no Brasil. In: LANDAU, Elena Charlotte.; MOURA, Larissa (Ed.). Variação geográfica do saneamento básico no Brasil em 2010: domicílios urbanos e rurais. Brasília: DF: Embrapa, p. 189-211, 2016.

MOREIRA, Mauricio Alves. Fundamentos do Sensoriamento Remoto e Metodologias de Aplicação. São José dos Campos: Instituto Nacional de Pesquisas Espaciais, 2001. 
NASCIMENTO, Leandro Silva; MORAES, Maria Letícia Marques. Avaliação da Distribuição Espacial de Quatro Doenças Infecciosas e Parasitárias no Município de Tomé-Açu/PA. 2019. Monografia (Graduação em Ciências Biológicas) - Faculdade de Ciências Biológicas, Universidade Federal Rural da Amazônia, Tomé-Açu, 2019.

PEREIRA, Maria Dias; LOPES, Junnia Duque; NEVES, Maria da Graça Camargo. Leishmaniose Visceral em Criança: um relato de caso sobre a recidiva da doença. Comunicação em Ciências da Saúde, v. 26, n.03/04, 2015. Disponível em: http://www.escs.edu.br/revistaccs/index.php/comunicacaoemcienciasdasaude/article/view/316 /143. Acesso em: 24 maio 2019.

PEREIRA, Rafael Alves; ALVES-SOUZA, Rosana Aparecida; VALE, Jéssica Sousa. O Processo de Transição Epidemiológica no Brasil: Uma Revisão de Literatura. Revista Científica da Faculdade de Educação e Meio Ambiente, n. 1, p. 99-108, 2015. Disponível: http://www.faema.edu.br/revistas/index.php/Revista-FAEMA/article/view/322/387. Acesso em: 24 maio 2019.

RODRIGUES, Aline De Freitas; ESCOBAR, Ana Lúcia; SOUSA-SANTOS, Reinaldo. Análise Espacial e Determinação de Áreas para o Controle da Malária no Estado de Rondônia. Revista da Sociedade Brasileira de Medicina Tropical, v. 41, n. 1, p. 55-64, 2008. Disponível em: https://www.scielo.br/scielo.php?script=sci_abstract\&pid=S0037$86822008000100011 \& \operatorname{lng}=$ pt\&nrm=iso. Acesso em: 19 jun. 2019.

SERPA, Hellen Dayane de Freitas. et al. Fatores Ambientais e Comportamentais do Homem Relacionados à Incidência de Casos de Leishmaniose Tegumentar Americana na Área de São José do Meriti, Ibatiba-ES. In: V SEMINÁRIO CIENTÍFICO DO UNIFACIG, 5., 2019, Manhuaçu. Anais [...]. Minas Gerais: UNIFAG, 2017. Disponível em: http://www.pensaracademico.facig.edu.br/index.php/semiariocientifico/article/view/1190/109 9. Acesso em: 19 jun. 2019.

SILVA, Brenda Caroline Sampaio et al. Análise Espacial da Leishmaniose Visceral no Município de Abaetetuba/PA, no período de 2007 a 2015. Associação Brasileira de Engenharia Sanitária e Ambiental, n. 1, p. 1-9, 2017. Disponível em: https://patua.iec.gov.br/bitstream/handle/iec/3681/An\%c3\%a1lise\%20espacial\%20da\%20leis hmaniose \%20visceral\%20no\%20munic\%c3\%adpio\%20de\%20Abaetetuba/Pa\%2c\%20no\%20 per\%c3\%adodo\%20de\%202007\%20a\%202015.pdf?sequence=1\&isAllowed=y. Acesso em: 19 jun. 2019.

SILVA, Richard Marques da et al. Modelagem Geoespacial e Temporal da Hanseníase entre 2001 e 2011 no Município de Bayeux, Paraíba. Revista Brasileira de Geografia Médica e da Saúde -, v. 8, n. 1, p. 89-103, dez. 2012. Disponível em: https://www.scielo.br/scielo.php?script=sci_arttext\&pid=S1982-45132012000200013. Acesso em: 19 jun. 2019.

SILVEIRA, Fernando Tobias. et al. Revisão Sobre a Patogenia da Leishmaniose Tegumentar Americana na Amazônia, com Ênfase à Doença Causada por Leishmania (V.) braziliensis e Leishmania (L.) amazonensis. Revista Paraense de Medicina, Belém, n. 1, mar. 2008. Disponível em: http://scielo.iec.gov.br/scielo.php?pid=S0101 59072008000100002\&script=sci_arttext\&tlng=en. Acesso em: 19 jun. 2019. 
SILVERMAN, Bernard Walter. Density Estimation for Statistic and Data Analysis. New York: Chapman and Hall, 1986.

SKABA, Daniel Albert et al. Geoprocessamento dos dados da saúde: o tratamento dos endereços. Cadernos de Saúde Pública, Rio de Janeiro, v. 20, p. 1753-1756, nov./dez. 2004.Disponível em: https://www.scielo.br/scielo.php?pid=S0102-

311X2004000600037\&script=sci_abstract\&tlng=pt. Acesso em: 19 jun. 2019.

VASCONCELOS, Cintia Honório et al. Uso do Sensoriamento Remoto para Estudar a Influência de Alterações Ambientais na Distribuição da Malária na Amazônia Brasileira.

Caderno Saúde Pública, Rio de Janeiro, v. 22, n. 3, p. 517-526, mar. 2006. Disponível em: https://www.scielo.br/scielo.php?pid=S0102-

311X2006000300006\&script=sci_abstract\&tlng=pt. Acesso em: 19 jun. 2019.

WERNECK, Guilherme Loureiro. Fórum: Geographic Spread and Urbanization of Visceral Leishmaniasis in Brazil. Caderno de Saúde Pública, Rio de Janeiro, n. 12, dez. 2008.

Disponível em: https://www.scielo.br/scielo.php?pid=S0102-

311X2008001200023\&script=sci_arttext. Acesso em: 19 jun. 2019.

XIMENES, Ricardo Arraes de Alencar et al. Vigilância de doenças endêmicas em áreas urbanas: a interface entre mapas de setores censitários e indicadores de morbidade. Caderno de Saúde Pública, Rio de Janeiro, n.1, jan.-mar. 1999. Disponível em: https://www.scielo.br/pdf/csp/v15n1/0035.pdf. Acesso em: 19 jun. 2019. 